\title{
Quantitative iTRAQ LC-MS/MS Proteomics Reveals the Proteome Profiles of DF-1 Cells after Infection with Subgroup J Avian Leukosis Virus
}

\author{
Xiaofei Li, ${ }^{1}$ Qi Wang, ${ }^{1}$ Yanni Gao, ${ }^{1}$ Xiaole Qi, ${ }^{1}$ Yongqiang Wang, ${ }^{1}$ Honglei Gao, \\ Yulong Gao, ${ }^{1}$ and Xiaomei Wang ${ }^{1,2}$ \\ ${ }^{1}$ Division of Avian Infectious Diseases, State Key Laboratory of Veterinary Biotechnology, Harbin Veterinary Research Institute, \\ Chinese Academy of Agricultural Sciences, Harbin 150001, China \\ ${ }^{2}$ Jiangsu Co-Innovation Center for Prevention and Control of Important Animal Infectious Disease and Zoonoses, \\ Yangzhou 225009, China
}

Correspondence should be addressed to Yulong Gao; ylg@hvri.ac.cn and Xiaomei Wang; xmw@hvri.ac.cn

Received 7 August 2014; Revised 9 December 2014; Accepted 17 December 2014

Academic Editor: Michele Rechia Fighera

Copyright (C) 2015 Xiaofei Li et al. This is an open access article distributed under the Creative Commons Attribution License, which permits unrestricted use, distribution, and reproduction in any medium, provided the original work is properly cited.

\begin{abstract}
Avian leukosis virus subgroup J (ALV-J) is an avian oncogenic retrovirus that can induce various clinical tumors and has caused severe economic losses in China. To improve our understanding of the host cellular responses to virus infection and the pathogenesis of ALV-J infection, we applied isobaric tags for relative and absolute quantification (iTRAQ) labeling coupled with multidimensional liquid chromatography-tandem mass spectrometry to detect the protein changes in DF-1 cells infected and mock-infected with ALV-J. A total of 75 cellular proteins were significantly changed, including 33 upregulated proteins and 42 downregulated proteins. The reliability of iTRAQ-LC MS/MS was confirmed via real-time PCR. Most of these proteins were related to the physiological functions of metabolic processes, biosynthetic processes, responses to stimuli, protein binding, signal transduction, cell cytoskeleton, and so forth. We also found some proteins that play important roles in apoptosis and oncogenicity. The differentially expressed proteins identified may provide valuable information to elucidate the pathogenesis of virus infection and virus-host interactions.
\end{abstract}

\section{Introduction}

The J subgroup of avian leukosis virus (ALV-J), which belongs to the Retroviridae family, was first isolated from whitemeat-type chickens in the United Kingdom in 1988 [1]. It can predominantly lead to myeloid leukosis (ML) and immunosuppression effects in both naturally and experimentally infected chickens $[2,3]$. In China, ALV-J-associated myeloid leukosis in chickens was first reported in 1999 [4]. ALV-J can induce various tumors, growth retardation, and production problems. In addition, in recent years, it has become widespread in many parts of our country and leads to severe economic losses in the poultry industry.

The pathogenesis of virus infection and the mechanism through which the virus interacts with host cells remain unclear. During virus infection, the proteins of host cells may be significantly changed. It is now possible to use proteomic techniques to identify the changes in protein abundance that indicate host cellular responses to virus infection and provide useful information to obtain a better understanding of the pathogenesis of virus infection [5-8]. Kvaratskhelia et al. [9] applied enzymatic digestion coupled with mass spectrometry (MS) to detect the sites of glycosylation on the surface of avian leukosis virus subgroup A (ALV-A) and found that carbohydrates may play an important role in receptor binding.

To explore the possible mechanisms of virus infection, we used isobaric tags for relative and absolute quantification (iTRAQ) combined with multidimensional liquid chromatography (LC) and tandem MS analysis to perform a 
quantitative proteomic analysis of DF-1 cells infected with ALV-J [10]. To the best of our knowledge, no previous study had used the iTRAQ LC-MS/MS proteomics strategy to investigate the differently expressed proteins in ALV-Jinfected DF-1 cells. The iTRAQ labeling technology could greatly increase the identification sensitivity and quantitation accuracy of proteomic analyses through a multiplexed quantitation strategy [11]. The results showed that 75 proteins were significantly changed after ALV-J infection. These changed proteins may provide valuable information to study the molecular mechanisms underlying ALV-J pathogenesis.

\section{Materials and Methods}

2.1. Reagents. The iTRAQ Reagent Multi-Plex Kit was acquired from Applied Biosystems (Foster City, CA, USA). A multidimensional liquid chromatographer (RIGOL 3220) was purchased from RIGOL, and the chromatographic column (Agela, C18 chromatographic column, $250 \times 4.6 \mathrm{~mm}$ i.d., filler particles diameter: $5 \mu \mathrm{m}$ ) was acquired from Agela Co., Ltd. (Tianjin, China). The LC-MS/MS instrument (QExactive) was obtained from Thermo Fisher Scientific.

2.2. Cell Culture and Virus Infection. DF-1 cells (ATCC accession number: CRL-12203) were cultured in Dulbecco's modified Eagle medium (DMEM; HyClone, Beijing, China) supplemented with $10 \%$ fetal bovine serum (FBS) and $100 \mu \mathrm{g} / \mathrm{mL}$ streptomycin and penicillin at $37^{\circ} \mathrm{C}$ in a $5 \% \mathrm{CO}_{2}$ atmosphere. ALV-J strain HPRS-103 (GenBank: Z46390) was kindly provided by Professor Venugopal Nair. DF-1 cells cultured in flasks to approximately $80 \%$ confluence were infected with $0.5 \mathrm{~mL}$ of $10^{3.5} / \mathrm{mL} 50 \%$ tissue culture infectious doses (TCID50) of ALV-J for $144 \mathrm{~h}$. Uninfected DF-1 cells served as mock-infected cells.

2.3. Indirect Immune Fluorescence Assay (IFA). At $144 \mathrm{~h}$ after infection, the infected DF-1 cells were washed twice with PBS and fixed with anhydrous ethanol for $20 \mathrm{~min}$. The fixed cells were then incubated with mouse anti-P27 monoclonal antibody (prepared in our lab) at $37^{\circ} \mathrm{C}$ for $60 \mathrm{~min}$. After washing three times with PBST (0.01 M PBS, pH 7.2, 0.05\% Tween 20), the cells were incubated with goat anti-mouse IgG conjugated to FITC (Sigma, USA) at $37^{\circ} \mathrm{C}$ for another $60 \mathrm{~min}$. Finally, the cells were observed under a Carl Zeiss Vision microscope (ZEISS Axio Observer D1) after three washes with PBST.

2.4. Protein Extraction, Digestion, and Labeling with iTRAQ Reagents. Infected and mock-infected DF-1 cells were washed twice with PBS. The cells were lysed in a lysis buffer (9 M urea, 4\% CHAPS, 1\% DTT, and 1\% IPG buffer). The mixtures were centrifuged at $15,000 \mathrm{~g}$ and $4^{\circ} \mathrm{C}$ for $15 \mathrm{~min}$. The supernatant was collected, and the protein concentration was determined using the Bradford protein assay [12] (Bio-Rad Laboratories). Then, $100 \mu \mathrm{g}$ of protein was mixed overnight with four volumes of cold $\left(-20^{\circ} \mathrm{C}\right)$ acetone and then dissolved using the dissolution buffer. After being reduced, alkylated, and digested with trypsin, the samples were labeled following the manufacturer's instructions
TABLE 1

\begin{tabular}{llllll}
\hline Time & 0 & 24 & 30 & 31 & 38 \\
\hline $\mathrm{B} \%$ & 3 & 16 & 30 & 90 & 90 \\
\hline
\end{tabular}

described in the iTRAQ protocol. The labeled samples were pooled for further analysis.

2.5. LC-MS/MS and Database Searches. The iTRAQ-labeled sample mixtures were then fractionated by strong cation exchange (SCX) chromatography on a high-performance liquid chromatography (HPLC) system (RIGOL 3220; Beijing, China) using a chromatographic column (Agela, C18 chromatographic column, $250 \times 4.6 \mathrm{~mm}$ i.d., filler particles diameter: $5 \mu \mathrm{m}$; Tianjin, China). Mobile phase A consisted of $2 \% \mathrm{ACN}-98 \% \mathrm{H}_{2} \mathrm{O}(\mathrm{pH} 10.0)$, and mobile phase B consisted of $98 \% \mathrm{ACN}-2 \% \mathrm{H}_{2} \mathrm{O}(\mathrm{pH} 10.0)$. The solvent gradient was as follows: $5 \%-8 \% \mathrm{~B}$ for $1 \mathrm{~min}, 8 \%-32 \% \mathrm{~B}$ for $24 \mathrm{~min}, 32 \%-$ $95 \%$ B for $2 \mathrm{~min}, 95 \%$ for $4 \mathrm{~min}$, and $95 \%-5 \%$ B for $1 \mathrm{~min}$. The column temperature was $45^{\circ} \mathrm{C}$, the flow rate was $0.7 \mathrm{~mL} / \mathrm{min}$, and the detection wavelength was $214 \mathrm{~nm}$. Peptides were collected every minute within the effective gradient from $8 \%$ to $32 \%$. A total of 27 fractions were collected and then dried.

The dried fractions were dissolved in 1.9\% ACN/98\% $\mathrm{H}_{2} \mathrm{O} / 0.1 \%$ FA aqueous solution and combined into nine samples. The samples were centrifuged at $12,000 \times \mathrm{r}$ for $3 \mathrm{~min}$, and the supernatant was collected. The supernatant was then analyzed using the EASY-nLC-1000 liquid phase interfaced with a Q Exactive mass spectrometer (Thermo Fisher). The chromatographic conditions are as follows: liquid phase, EASY-nLC-1000; enriching column, C18, $5 \mu \mathrm{m}$, ID100 $\mu \mathrm{m}$, $20 \mathrm{~mm}$ in length; separation column, C18, $3 \mu \mathrm{m}$, ID75 $\mu \mathrm{m}$, $120 \mathrm{~mm}$ in length; mobile phase $\mathrm{A}, 1.9 \% \mathrm{ACN}+98 \% \mathrm{H}_{2} \mathrm{O}+$ $0.1 \%$ FA; mobile phase $\mathrm{B}, 98 \% \mathrm{ACN}+1.9 \% \mathrm{H}_{2} \mathrm{O}+0.1 \% \mathrm{FA}$; and flow rate, $450 \mathrm{nl} / \mathrm{min}$.

\section{Elution Conditions. See Table 1.}

The data were acquired at $38 \mathrm{~min}$. The spray voltage was 2.0 KV, the capillary temperature was $320^{\circ} \mathrm{C}$, the collision energy was 30 , and the acquisition quality range was $300-$ 1400 da.

The relative quantification and protein identification were performed with the Protein Discoverer software (version 1.2) using the built-in mascot as the search engine.

2.6. Real-Time PCR. The primers (Table 2) were synthesized by BoShi Biotechnology Company (Harbin, China). The gene was amplified from the genomic DNA of DF-1 cells by polymerase chain reaction (PCR). The PCR-amplified products were separated in a $2 \%$ agarose gel and then purified using a DNA gel extraction kit (Axygen Biotechnology Limited, Hangzhou City, China). The products were then ligated into the pZeroBack/blunt vector (Tiangen Biotech Co., Ltd., Beijing, China), and the sequence was verified. The plasmid DNA was used as the standard to construct the standard curve via SYBR Green real-time PCR. The total cellular RNA of the infected or mock-infected DF-1 cells was extracted using the RNeasy Mini Kit (QIAGEN, China) 
TABLE 2: Primer sequences for real-time PCR.

\begin{tabular}{lcc}
\hline Gene & \multicolumn{1}{c}{ Sequence } & Size \\
\hline \multirow{2}{*}{ BLOC1S5 } & $\begin{array}{r}\text { F-TATATGAGCGGGGCAGGCCCT } \\
\text { R-TTCCCCGACATCCTTGAT }\end{array}$ & $150 \mathrm{bp}$ \\
\hline \multirow{2}{*}{ Keratin } & $\begin{array}{l}\text { F-ATGTCCCGCTCCGTCAGCTTC } \\
\text { R-AGAGCCCAGGTTGTAGAGGCT }\end{array}$ & $150 \mathrm{bp}$ \\
\hline \multirow{2}{*}{ HMG14 } & $\begin{array}{l}\text { F-ATGCCGAAGAGAAAGGTG } \\
\text { R-TCAGATTTATCCTTAGCCGCC }\end{array}$ & $140 \mathrm{bp}$ \\
\hline \multirow{2}{*}{ AACS } & $\begin{array}{l}\text { F-ATGTCCCGCGAGCCCGAGATT } \\
\text { R-CACTGACCACTGGTATAAGTC }\end{array}$ & $150 \mathrm{bp}$ \\
\hline
\end{tabular}

according to the manufacturer's protocol. Reverse transcription was performed using a PrimeScript II First-Strand cDNA Synthesis Kit (TaKaRa, China) as described in the protocol. The real-time PCR was performed using the Roche LightCycler 480 real-time PCR System.

2.7. Bioinformatics Analysis. The functional annotation of the 75 proteins in DF-1 cells that were significantly changed after infection with ALV-J was performed using the GOSlimViewer tool of the AgBase database (http://www.agbase.msstate.edu/) [13]. In addition, we aimed to determine how ALV-J interacts with the host cellular proteins and how it affects the function of host cells. The identified proteins were inputted into the STRING database to obtain the protein-protein interaction network $[14,15]$ (http://string.embl.de/).

\section{Results}

3.1. Confirmation of ALV-J Infection in DF-1 Cells by IFA. To confirm that the DF-1 cells were infected by ALV-J, IFA was used to detect the viral P27 antigen. The results showed clear green fluorescence in ALV-J-infected DF-1 cells $144 \mathrm{~h}$ after infection, whereas the uninfected DF-1 cells exhibited no green fluorescence (Figure 1).

\subsection{Protein Profile Obtained by iTRAQ LC-MS/MS Analysis.} To explore the differences in the protein expression levels after virus infection, the total proteins of ALV-J-infected and mock-infected DF-1 cells were extracted for iTRAQLC-MS/MS analysis. A total of 1091 proteins were detected, including 75 proteins in DF-1 cells that were significantly changed infection with ALV-J for $144 \mathrm{~h}$ (Table 3). These differently expressed proteins were divided into two clusters: upregulated and downregulated. The number of upregulated proteins was 33, whereas the number of downregulated proteins was 42 .

3.3. Functional Classifications of the Identified Proteins. To annotate the functions of the 75 significantly changed proteins identified in our study, the proteins were submitted to GORetriever (http://www.agbase.msstate.edu/) for analysis. Three types of annotations were obtained using the website: molecular functions, biological processes, and cellular components.
The biological process annotation revealed that the significantly changed proteins were involved in metabolic process (19\%), macromolecule metabolic process $(12 \%)$, regulation of biological process (11\%), biosynthetic processes $(10 \%)$, nucleobase-containing compound metabolic process $(10 \%)$, response to stimulus (7\%), and various other activities (31\%) (Figure 2, biological process).

The molecular function annotation revealed that these differently expressed proteins were involved in protein binding (30\%), nucleic acid binding (21\%), hydrolase activity (11\%), transferase activity (5\%), receptor activity (5\%), oxidoreductase activity (4\%), and various other activities (24\%) (Figure 2, molecular function).

The cellular component annotation revealed that the altered proteins were associated with the following cellular components: intracellular (28\%), cytoplasm (24\%), nucleus $(17 \%)$, membrane $(15 \%)$, extracellular region $(5 \%)$, chromosome $(3 \%)$, and various others $(8 \%)$ (Figure 2 , cellular component).

3.4. Validation of the iTRAQ Data by Real-Time PCR. To confirm the results of the differentially expressed proteins identified by iTRAQ LC-MS/MS analysis, real-time PCR was performed to detect the transcript expression levels of the genes after ALV-J infection. We generated four standard curves to determine the gene expression of BLOC1S5, keratin, HMG14, and AACS in ALV-J-infected and mock-infected DF-1 cells. The results showed that HMG14 was upregulated (Figure 3), whereas BLOC1S5, AACS, and keratin were downregulated (Figure 3). The RT-PCR results were consistent with the results of the iTRAQ LC-MS/MS analysis (Table 3), confirming that the iTRAQ data were reliable.

3.5. Protein-Protein Interaction Analysis. The mechanism through which the virus interacts with host cells remains unclear, and oncogenicity is an important index of the pathogenicity of ALV-J. During virus infection, some proteins of host cells may be significantly changed. As a result, the functions of the changed proteins will also be altered. In our study, we aimed to determine whether the significantly changed proteins that were identified have some relationship with apoptosis or ALV-J-induced oncogenicity. We searched the STRING database to analyze the protein-protein interactions between the differently expressed proteins and PARK7, PTENP1, AKT1, PIK3CA (PI3K), and VDAC (Figure 4). These proteins are known to have some relationship with tumor-associated process and apoptosis. The protein-protein interaction networks may provide valuable information to further investigate the possible mechanism of ALV-J-induced oncogenicity.

\section{Discussion}

Proteomics is a relatively novel technology that has been used for the detection of the host cellular proteins response to virus infection $[16,17]$. Isobaric tags for relative and absolute quantification (iTRAQ) combined with multidimensional liquid chromatography (LC) and tandem MS analysis are a powerful tool for quantitative proteomic analysis that has 


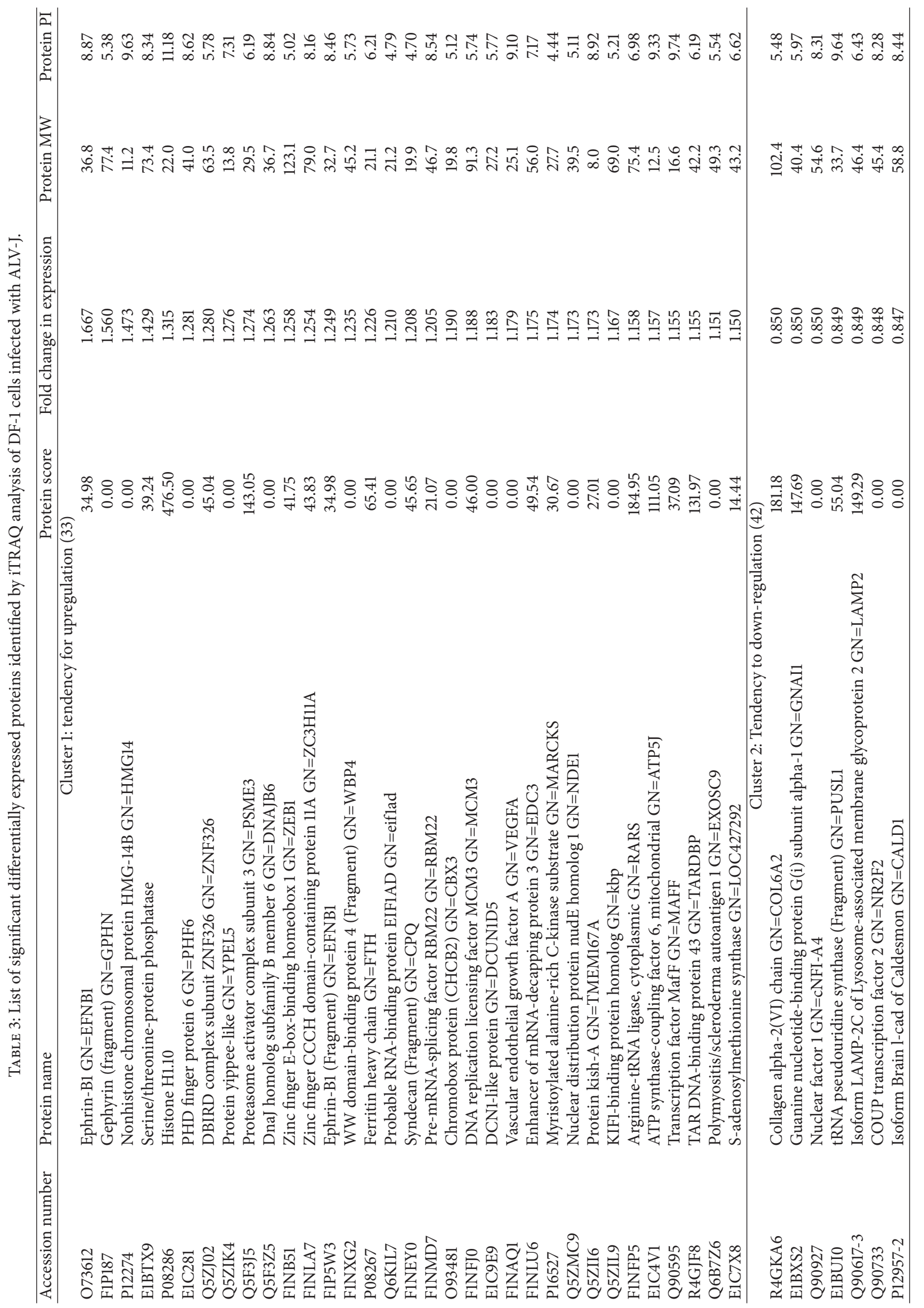




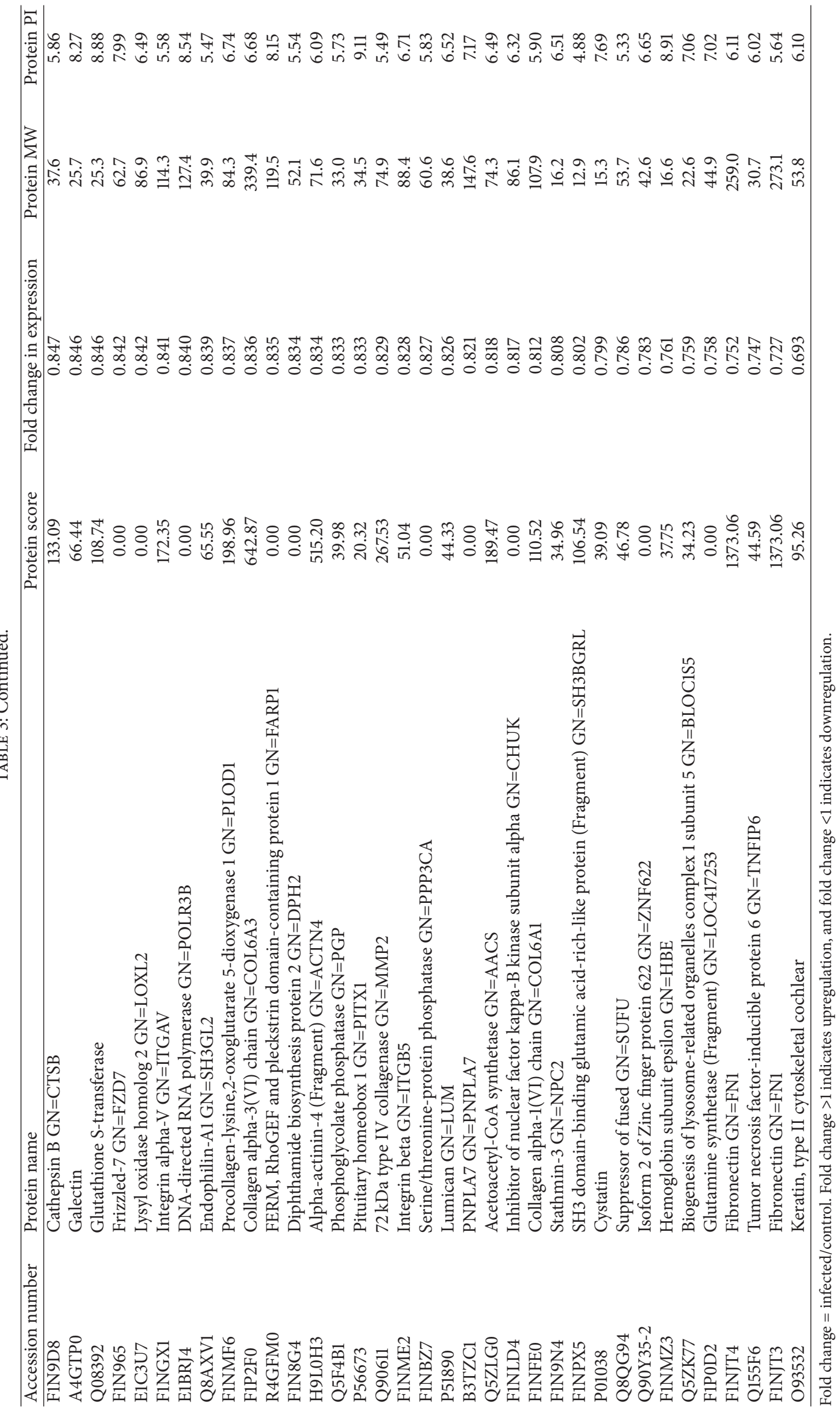




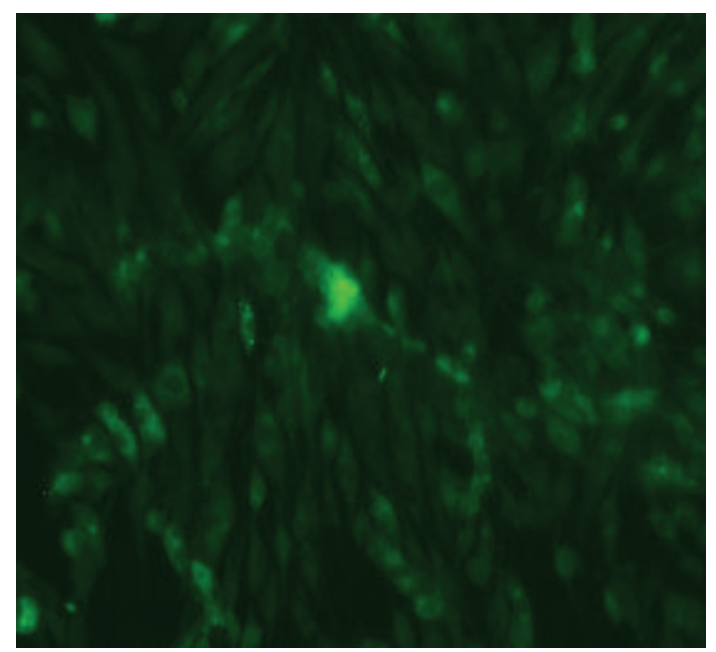

(a)

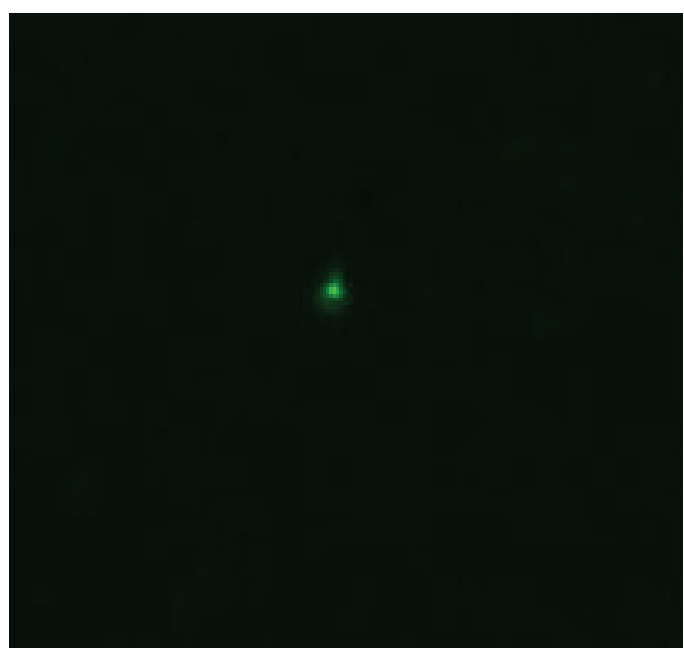

(b)

Figure 1: Identification of DF-1 cells infected with ALV-J by IFA. (a) DF1 cells infected with ALV-B. (b) Normal uninfected DF1 cells.

been widely applied in many studies [18-20]. In this study, we first applied the iTRAQ approach to identify the differential protein expression profiles of DF-1 cells infected with ALVJ. Using the iTRAQ LC-MS/MS technology, the significantly changed proteins were mostly associated with metabolic process, signal transducer activity, cell cytoskeleton, oxidoreductase activity, response to stimulus, and immune responses. In addition, some apoptosis and tumor-associated proteins (VEGF-A, ACTN4, and METAP2) were also identified by the iTRAQ LC-MS/MS technology.

4.1. Alterations of Tumor-Associated Proteins. Vascular endothelial growth factor A (VEGF-A) is an important inducer of angiogenesis [21]. As has been shown in many reports, upregulated VEGF-A can induce tumor formation via some unique signaling pathways $[22,23]$. In addition, VEGF-A, which is known as a positive regulator, contributes to tumor growth and promotes tumor formation [24, 25]. Previous studies described a threshold level of proteins to promote tumorigenesis, which indicated that the expression level of one protein needs to reach the threshold level before promoting tumorigenesis [26, 27]. Studies in our lab showed that the increased replication of ALV-J increased the expression of VEGF-A, indicating an increased opportunity for ALV-J to push the expression level of VEGF-A to reach the threshold level to promote tumorigenesis [27]. In this study, we found that VEGF-A is overexpressed in DF-1 cells after infection with ALV-J. The results further suggested that VEGF-A is closely associated with ALVJ-induced tumorigenesis and may also suggest a novel molecular mechanism for better understanding of the higher oncogenicity of ALV-J.

Alpha-actinins (ACTNs) were classified into cytoskeleton proteins, while ACTN4 has some other unique functions, such as signal transduction, protein expression regulation, and nuclear transport. Histological analyses of cancer tissues showed a strong correlation between ACTN4 expression and tumorigenesis in several types of cancers [28-30]. Furthermore, upregulated ACTN4 in cancer cells has been suggested as a biomarker for drug resistance and malignant cell invasion [31-34]. Previous studies showed that ALV-J infection in DF-1 cells led to rapid increase in Akt phosphorylation and the phosphorylation of Akt was PI3K-dependent [35]. $\mathrm{PI} 3 \mathrm{~K} / \mathrm{Akt}$ pathway also regulates viral replication of ALV-J [35]. Furthermore, AKT interacts with ACTN4 and ACTN4 is a functional partner of AKT [36]. Therefore, the upregulated ACTN4 observed in this study may be associated with tumorigenesis induced by ALV-J through PI3K/Akt pathway. This may provide useful information to elucidate the mechanism of ALV-J induced tumorigenesis and may also become potential therapeutic targets to control ALV-J infections.

METAP2 was considered to have some relationship with angiogenesis inhibition [37]. In addition, METAP2 can block B cell differentiation into plasma cells [38]. Some viruses, whose primary target cells are B cells, can clinically induce tumor formation. Therefore, downregulation of METAP2 in this study may influence the function of B cells, which may provide evidence to explain why ALV-J infection can result in immune suppression and tumorigenesis.

4.2. Redox Regulation. Peroxiredoxins (PRDXs), a family of peroxidases as antioxidant enzymes, can support tumor maintenance and survival through protecting cells from apoptosis induced by oxidative stress [39-41]. A previous study indicated that liver cells transfected with PRDX6 siRNA resulted in an increase in peroxide-induced cytotoxicity by apoptosis, which implies that decrease of PRDX6 promotes apoptosis [42]. Therefore, downregulated PRDX6 in this study suggests that ALV-J infection may weaken the antiapoptotic function of PRDX6.

In addition, PRDX1 was found to be upregulated in this study. A previous study indicated that the mice lacking 

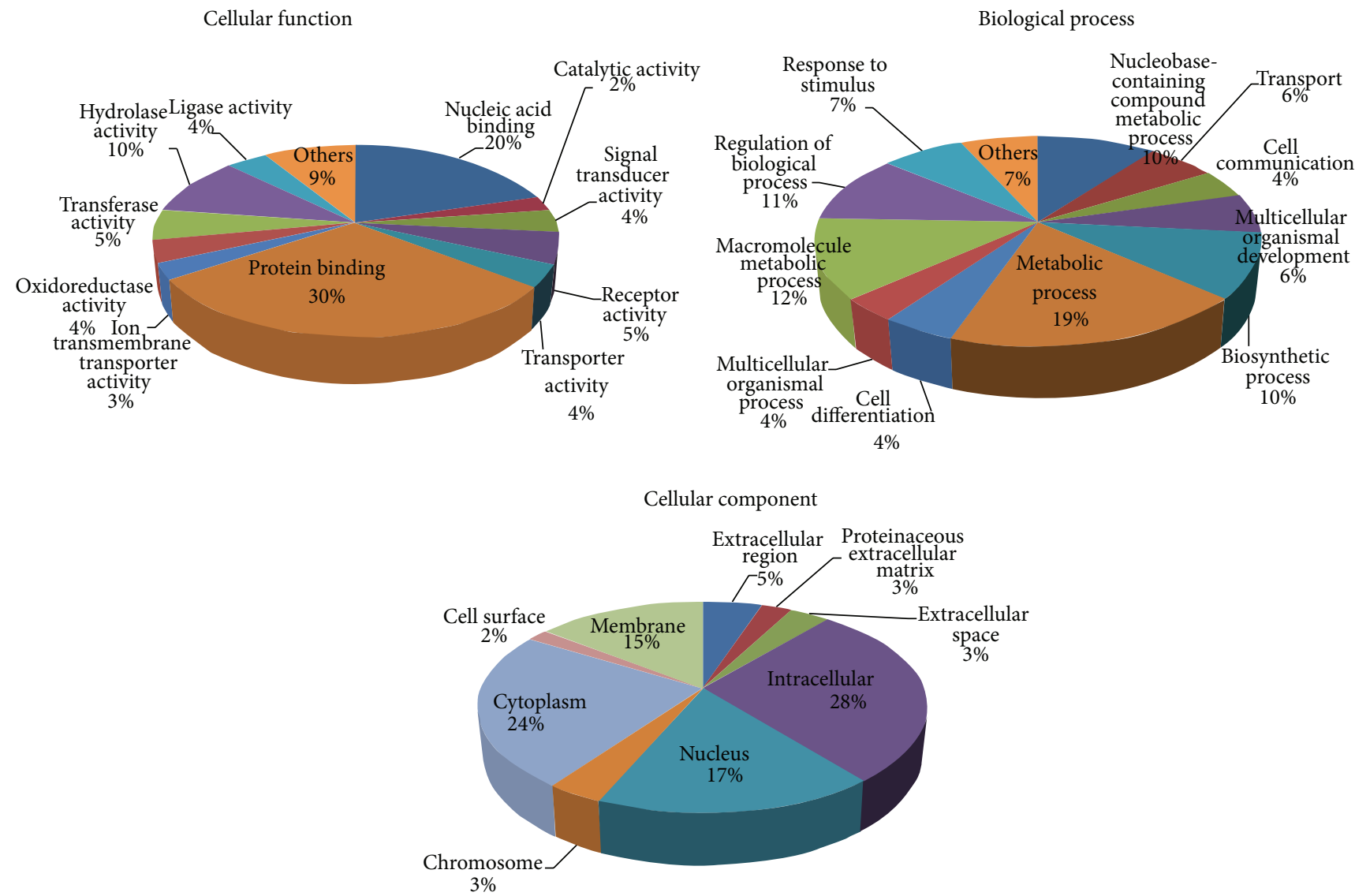

FIGURE 2: Functional annotation of the differently expressed proteins according to their biological process, molecular function, and cellular component.

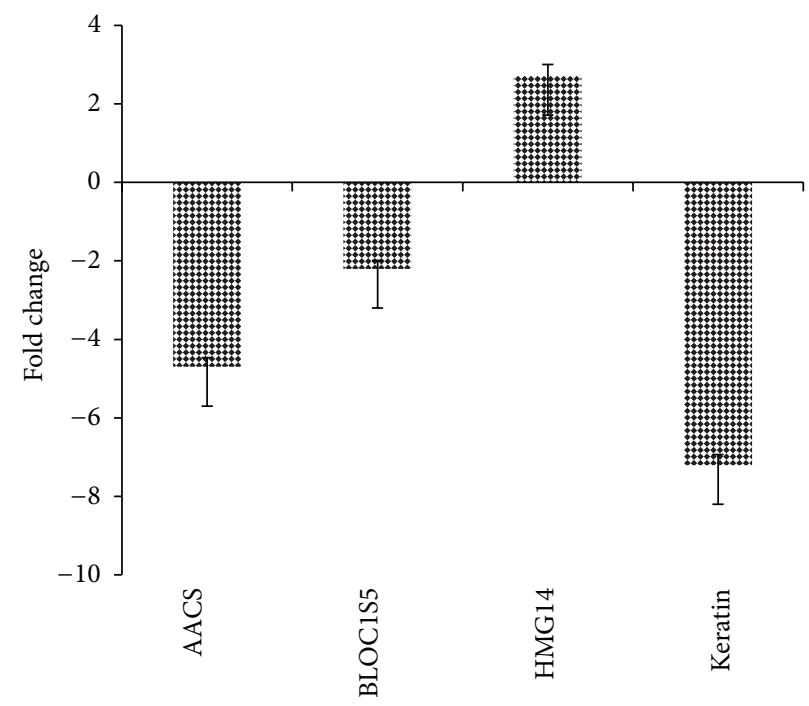

FIGURE 3: Transcriptional profiles of the significantly changed proteins in ALV-J-infected DF-1 cells. The error bars represent the standard deviations.

PRDX1 have several malignant cancers, including sarcomas, carcinomas, and lymphomas [43]. These malignancies are associated with low expression of PRDX1, which suggests that PRDX1 may function as a tumor suppressor [43]. Studies also indicated that PRDX1 interacts with the c-Myc oncogene and can inhibit its transcriptional activity [44] and high expression of PRDX1 appears to be associated with less aggressive breast cancers [45]. Therefore, upregulation of PRDX1 in this study may result from the defense of host cells responses to the ALV-J infection. 


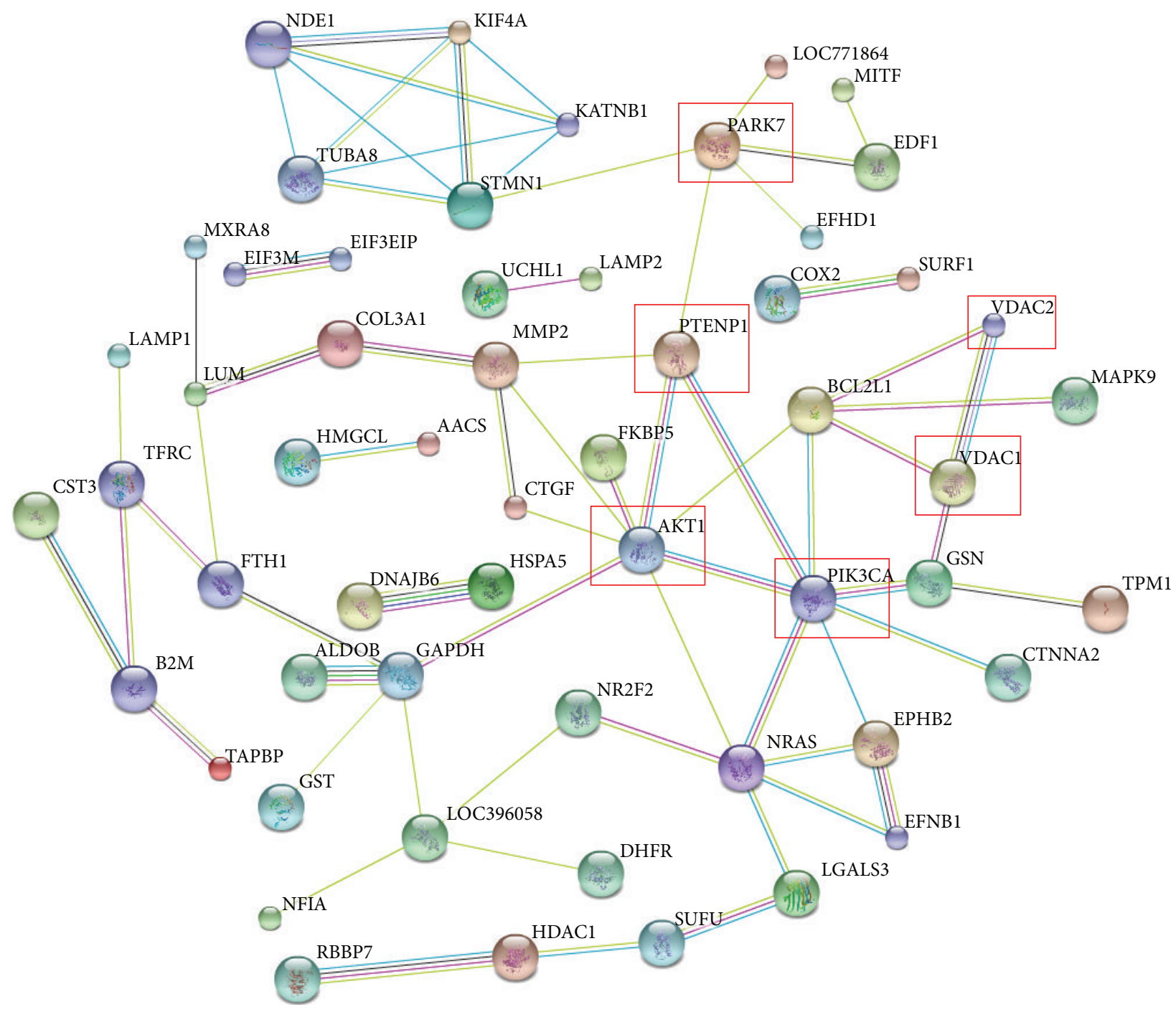

FIGURE 4: The protein-protein interaction between the identified proteins and the tumor- or apoptosis-associated proteins analyzed by the STRING software. An edge was drawn with up to seven differently colored lines, representing the existence of the seven types of evidence used for predicting the associations: a red line indicates the presence of fusion evidence; a yellow line indicates text mining evidence; a purple line indicates experimental evidence; a blue line indicates cooccurrence evidence; a light blue line indicates database evidence; a green line indicates neighborhood evidence; a black line indicates coexpression evidence.

4.3. Cytoskeleton Proteins and ALV-J Infection. Cytoskeleton proteins are involved in the maintenance of cell morphology, regulation of protein synthesis, endocytosis, cell movement, and cell-to-cell attachment $[46,47]$. As determined through iTRAQ LC-MS/MS analysis, some cytoskeleton proteins were identified to be significantly changed in DF-1 cells after infection with ALV-J. Isoform 2 of the F-actin-capping protein subunit beta isoforms 1 and 2 (CAPZB) can regulate the growth of actin filaments, and actin filaments play a vital role in the maintenance of cell morphology [48]. Furthermore, actin-related protein 3 (ACTR3) and actin-related protein 5 (ACTR5) were also found to be changed. The low expression of these proteins revealed that the cytoskeletal proteins were disrupted during infection with ALV-J. In addition, the differential expression of these proteins may be due to the interaction between the virus and host cellular proteins after infection with ALV-J.

It has been reported that keratins have become the standard detection marker for tumor cells and were also the most common marker to identify tumor cells [49]. Previous studies showed that before tumor cells got the ability to migrate and invade the host, they need to undergo epithelialmesenchymal transition, during which the cytoskeletons are rearranged and epithelial markers, such as keratins, claudins, and E-cadherin, are observed to be downregulated [49-52]. Immunohistochemical analysis showed that low expression of keratin was associated with a higher tumor grade in breast cancer [52]. Previous study indicated that acetoacetylCoA synthetase (AACS) was found in tumor tissues and plays important roles in metabolic processes of tumors [53]. 
Whether or not the downregulated keratin and AACS in this study were associated with tumorigenesis induced by ALV-J infection needs to be further investigated.

\section{Conclusions}

In summary, our study was the first to use iTRAQ LC-MS/MS to detect cellular responses to ALV-J infection in DF-1 cells. A total of 75 significantly changed proteins were identified. These differently expressed proteins may provide useful information for elucidating the molecular mechanism underlying the interaction between ALV-J and DF-1 cells and will also facilitate our understanding of the pathogenesis of ALV-J infection.

\section{Conflict of Interests}

The authors declare that they have no conflict of interests.

\section{Acknowledgments}

This study was funded by the National Natural Science Foundation of China (31372437 and 31201923) and the Earmarked Fund for the Modern Agro-industry Technology Research System (no. nycytx-42-G3-01).

\section{References}

[1] L. N. Payne, K. Howes, A. M. Gillespie, and L. M. Smith, "Host range of Rous sarcoma virus pseudotype RSV(HPRS-103) in 12 avian species: support for a new avian retrovirus envelope subgroup, designated J," Journal of General Virology, vol. 73, part 11, pp. 2995-2997, 1992.

[2] L. N. Payne, A. M. Gillespie, and K. Howes, "Recovery of acutely transforming viruses from myeloid leukosis induced by the HPRS-103 strain of avian leukosis virus," Avian Diseases, vol. 37, no. 2, pp. 438-450, 1993.

[3] R. L. Witter, "Avian tumor viruses: persistent and evolving pathogens," Acta Veterinaria Hungarica, vol. 45, no. 3, pp. 251266, 1997.

[4] Y. Du, Z. Cui, and A. Qin, "Subgroup J of avian leukosis viruses in China," China Poultry, vol. 3, pp. 1-4, 1999.

[5] R. Narayan, B. Gangadharan, O. Hantz et al., "Proteomic analysis of HepaRG cells: a novel cell line that supports hepatitis B virus infection," Journal of Proteome Research, vol. 8, no. 1, pp. 118-122, 2009.

[6] P. Alfonso, J. Rivera, B. Hernáez, C. Alonso, and J. M. Escribano, "Identification of cellular proteins modified in response to African swine fever virus infection by proteomics," Proteomics, vol. 4, no. 7, pp. 2037-2046, 2004.

[7] T. Toda, M. Sugimoto, A. Omori, T. Matsuzaki, Y. Furuichi, and N. Kimura, "Proteomic analysis of Epstein-Barr virustransformed human B- lymphoblastoid cell lines before and after immortalization," Electrophoresis, vol. 21, no. 9, pp. 1814$1822,2000$.

[8] J. Sun, Y. Jiang, Z. Shi et al., "Proteomic alteration of PK-15 cells after infection by classical swine fever virus," Journal of Proteome Research, vol. 7, no. 12, pp. 5263-5269, 2008.

[9] M. Kvaratskhelia, P. K. Clark, S. Hess, D. C. Melder, M. J. Federspiel, and S. H. Hughes, "Identification of glycosylation sites in the SU component of the Avian Sarcoma/Leukosis virus Envelope Glycoprotein (Subgroup A) by mass spectrometry," Virology, vol. 326, no. 1, pp. 171-181, 2004.

[10] P. L. Ross, Y. N. Huang, J. N. Marchese et al., "Multiplexed protein quantitation in Saccharomyces cerevisiae using aminereactive isobaric tagging reagents," Molecular and Cellular Proteomics, vol. 3, no. 12, pp. 1154-1169, 2004.

[11] K. Seggerson, L. Tang, and E. G. Moss, "Two genetic circuits repress the Caenorhabditis elegans heterochronic gene lin-28 after translation initiation," Developmental Biology, vol. 243, no. 2, pp. 215-225, 2002.

[12] M. M. Bradford, "A rapid and sensitive method for the quantitation of microgram quantities of protein utilizing the principle of protein-dye binding," Analytical Biochemistry, vol. 72, no. 1-2, pp. 248-254, 1976.

[13] J. Liu, J. Bai, Q. Lu et al., "Two-dimensional liquid chromatography-tandem mass spectrometry coupled with isobaric tags for relative and absolute quantification (iTRAQ) labeling approach revealed first proteome profiles of pulmonary alveolar macrophages infected with porcine circovirus type 2," Journal of Proteomics, vol. 79, pp. 72-86, 2013.

[14] N. Thanthrige-Don, M. F. Abdul-Careem, L. A. Shack, S. C. Burgess, and S. Sharif, "Analyses of the spleen proteome of chickens infected with Marek's disease virus," Virology, vol. 390, no. 2, pp. 356-367, 2009.

[15] Q. Lu, J. Bai, L. Zhang et al., “Two-dimensional liquid chromatography-tandem mass spectrometry coupled with isobaric tags for relative and absolute quantification (iTRAQ) labeling approach revealed first proteome profiles of pulmonary alveolar macrophages infected with porcine reproductive and respiratory syndrome virus," Journal of Proteome Research, vol. 11, no. 5, pp. 2890-2903, 2012.

[16] K. L. Maxwell and L. Frappier, "Viral proteomics," Microbiology and Molecular Biology Reviews, vol. 71, no. 2, pp. 398-411, 2007.

[17] X. Zheng, L. Hong, L. Shi, J. Guo, Z. Sun, and J. Zhou, "Proteomics analysis of host cells infected with infectious bursal disease virus," Molecular and Cellular Proteomics, vol. 7, no. 3, pp. 612-625, 2008.

[18] B. Seshi, "An integrated approach to mapping the proteome of the human bone marrow stromal cell," Proteomics, vol. 6, no. 19, pp. 5169-5182, 2006.

[19] H. Zhang, L. Lv, H. Liu et al., "Profiling the potential biomarkers for cell differentiation of pancreatic cancer using iTRAQ and 2D LC-MS/MS," Proteomics_Clinical Applications, vol. 3, no. 7, pp. 862-871, 2009.

[20] S. Muraoka, H. Kume, S. Watanabe et al., "Strategy for SRM-based verification of biomarker candidates discovered by iTRAQ method in limited breast cancer tissue samples," Journal of Proteome Research, vol. 11, no. 8, pp. 4201-4210, 2012.

[21] S. Takahashi, "Vascular endothelial growth factor (VEGF), VEGF receptors and their inhibitors for antiangiogenic tumor therapy," Biological and Pharmaceutical Bulletin, vol. 34, no. 12, pp. 1785-1788, 2011.

[22] A. Catalano, M. Romano, S. Martinotti, and A. Procopio, "Enhanced expression of vascular endothelial growth factor (VEGF) plays a critical role in the tumor progression potential induced by simian virus 40 large T antigen," Oncogene, vol. 21, no. 18, pp. 2896-2900, 2002.

[23] K. Ohtani, J. Usuda, S. Ichinose et al., "High expression of GADD-45alpha and VEGF induced tumor recurrence via upregulation of IL-2 after photodynamic therapy using NPe6," 
International Journal of Oncology, vol. 32, no. 2, pp. 397-403, 2008.

[24] E. Lorenzon, R. Colladel, E. Andreuzzi et al., "MULTIMERIN2 impairs tumor angiogenesis and growth by interfering with VEGF-A/VEGFR2 pathway," Oncogene, vol. 31, no. 26, pp. 31363147, 2012.

[25] N. Oka, A. Soeda, A. Inagaki et al., "VEGF promotes tumorigenesis and angiogenesis of human glioblastoma stem cells," Biochemical and Biophysical Research Communications, vol. 360, no. 3, pp. 553-559, 2007.

[26] Q. Li, T.-O. Ishikawa, M. Oshima, and M. M. Taketo, “The threshold level of adenomatous polyposis coli protein for mouse intestinal tumorigenesis," Cancer Research, vol. 65, no. 19, pp. 8622-8627, 2005.

[27] Q. Wang, Y. Gao, Y. Wang et al., "A 205-nucleotide deletion in the 3' untranslated region of avian leukosis virus subgroup J, currently emergent in China, contributes to its pathogenicity," Journal of Virology, vol. 86, no. 23, pp. 12849-12860, 2012.

[28] K. Honda, T. Yamada, Y. Hayashida et al., "Actinin-4 increases cell motility and promotes lymph node metastasis of colorectal cancer," Gastroenterology, vol. 128, no. 1, pp. 51-62, 2005.

[29] T. Welsch, S. Keleg, F. Bergmann, S. Bauer, U. Hinz, and J. Schmidt, "Actinin-4 expression in primary and metastasized pancreatic ductal adenocarcinoma," Pancreas, vol. 38, no. 8, pp. 968-976, 2009.

[30] S. Yamamoto, H. Tsuda, K. Honda et al., "Actinin-4 expression in ovarian cancer: a novel prognostic indicator independent of clinical stage and histological type," Modern Pathology, vol. 20, no. 12, pp. 1278-1285, 2007.

[31] J. Fellenberg, M. J. Dechant, V. Ewerbeck, and H. Mau, "Identification of drug-regulated genes in osteosarcoma cells," International Journal of Cancer, vol. 105, no. 5, pp. 636-643, 2003.

[32] J. He, S. A. Whelan, M. Lu et al., "Proteomic-based biosignatures in breast cancer classification and prediction of therapeutic response," International Journal of Proteomics, vol. 2011, Article ID 896476, 16 pages, 2011.

[33] S. Kikuchi, K. Honda, H. Tsuda et al., "Expression and gene amplification of actinin-4 in invasive ductal carcinoma of the pancreas," Clinical Cancer Research, vol. 14, no. 17, pp. 53485356, 2008.

[34] C. Zhou, Q. Zhong, L. V. Rhodes et al., "Proteomic analysis of acquired tamoxifen resistance in MCF-7 cells reveals expression signatures associated with enhanced migration," Breast Cancer Research, vol. 14, no. 2, article R45, 2012.

[35] S.-Z. Feng, W.-S. Cao, and M. Liao, "The PI3K/Akt pathway is involved in early infection of some exogenous avian leukosis viruses," Journal of General Virology, vol. 92, no. 7, pp. 1688-1697, 2011.

[36] Z. Ding, J. Liang, Y. Lu et al., "A retrovirus-based protein complementation assay screen reveals functional AKT1-binding partners," Proceedings of the National Academy of Sciences of the United States of America, vol. 103, no. 41, pp. 15014-15019, 2006.

[37] P. Selvakumar, A. Lakshmikuttyamma, J. R. Dimmock, and R. K. Sharma, "Methionine aminopeptidase 2 and cancer," Biochimica et Biophysica Acta-Reviews on Cancer, vol. 1765, no. 2, pp. 148-154, 2006.

[38] R. C. Priest, J. Spaull, J. Buckton et al., "Immunomodulatory activity of a methionine aminopeptidase- 2 inhibitor on B cell differentiation," Clinical and Experimental Immunology, vol. 155, no. 3, pp. 514-522, 2009.
[39] Y. K. So, H. Y. Jo, H. K. Mi et al., " $\mathrm{H}_{2} \mathrm{O}_{2}$-dependent hyperoxidation of peroxiredoxin $6(\operatorname{Prdx} 6)$ plays a role in cellular toxicity via up-regulation of iPLA2 activity," Journal of Biological Chemistry, vol. 283, no. 48, pp. 33563-33568, 2008.

[40] N. Fatma, E. Kubo, M. Sen et al., "Peroxiredoxin 6 delivery attenuates TNF-alpha-and glutamate-induced retinal ganglion cell death by limiting ROS levels and maintaining $\mathrm{Ca}^{2+}$ homeostasis," Brain Research, vol. 1233, pp. 63-78, 2008.

[41] C. A. Neumann and Q. Fang, "Are peroxiredoxins tumor suppressors?” Current Opinion in Pharmacology, vol. 7, no. 4, pp. 375-380, 2007.

[42] B. Walsh, A. Pearl, S. Suchy, J. Tartaglio, K. Visco, and S. A. Phelan, "Overexpression of Prdx6 and resistance to peroxideinduced death in Hepal-6 cells: Prdx suppression increases apoptosis," Redox Report, vol. 14, no. 6, pp. 275-284, 2009.

[43] C. A. Neumann, D. S. Krause, C. V. Carman et al., "Essential role for the peroxiredoxin Prdxl in erythrocyte antioxidant defence and tumour suppression," Nature, vol. 424, no. 6948, pp. 561565, 2003.

[44] R. A. Egler, E. Fernandes, K. Rothermund et al., "Regulation of reactive oxygen species, DNA damage, and c-Myc function by peroxiredoxin 1," Oncogene, vol. 24, no. 54, pp. 8038-8050, 2005.

[45] E.-H. L. Pei-Jou Chua, G. Chunhua, W.-C. Y. George, T. PuayHoon, and B. Boon-Huat, "Clinicopathological correlation of peroxiredoxin I in breast cancer," in Proceedings of the World Medical Conference, Prague, Czech Republic, 2011.

[46] M. Radulovic and J. Godovac-Zimmermann, "Proteomic approaches to understanding the role of the cytoskeleton in host-defense mechanisms," Expert Review of Proteomics, vol. 8, no. 1, pp. 117-126, 2011.

[47] W.Zou, J. Ke, A. Zhang et al., "Proteomics analysis of differential expression of chicken brain tissue proteins in response to the neurovirulent $\mathrm{H} 5 \mathrm{~N} 1$ avian influenza virus infection," Journal of Proteome Research, vol. 9, no. 8, pp. 3789-3798, 2010.

[48] J. F. Casella and D. J. Maack, "Purification and initial characterization of a protein from skeletal muscle that caps the barbed ends of actin filaments," The Journal of Biological Chemistry, vol. 261, no. 23, pp. 10915-10921, 1986.

[49] S. A. Joosse, J. Hannemann, J. Spötter et al., "Changes in keratin expression during metastatic progression of breast cancer: impact on the detection of circulating tumor cells," Clinical Cancer Research, vol. 18, no. 4, pp. 993-1003, 2012.

[50] J. P. Thiery and J. P. Sleeman, "Complex networks orchestrate epithelial-mesenchymal transitions," Nature Reviews Molecular Cell Biology, vol. 7, no. 2, pp. 131-142, 2006.

[51] D. C. Radisky, "Epithellial-mesenchymal transition," Journal of Cell Science, vol. 118, no. 19, pp. 4325-4326, 2005.

[52] B. Willipinski-Stapelfeldt, S. Riethdorf, V. Assmann et al., "Changes in cytoskeletal protein composition indicative of an epithelial-mesenchymal transition in human micrometastatic and primary breast carcinoma cells," Clinical Cancer Research, vol. 11, no. 22, pp. 8006-8014, 2005.

[53] M. J. Tisdale, "Role of acetoacetyl-CoA synthetase in acetoacetate utilization by tumor cells," Cancer Biochemistry Biophysics, vol. 7, no. 2, pp. 101-107, 1984. 

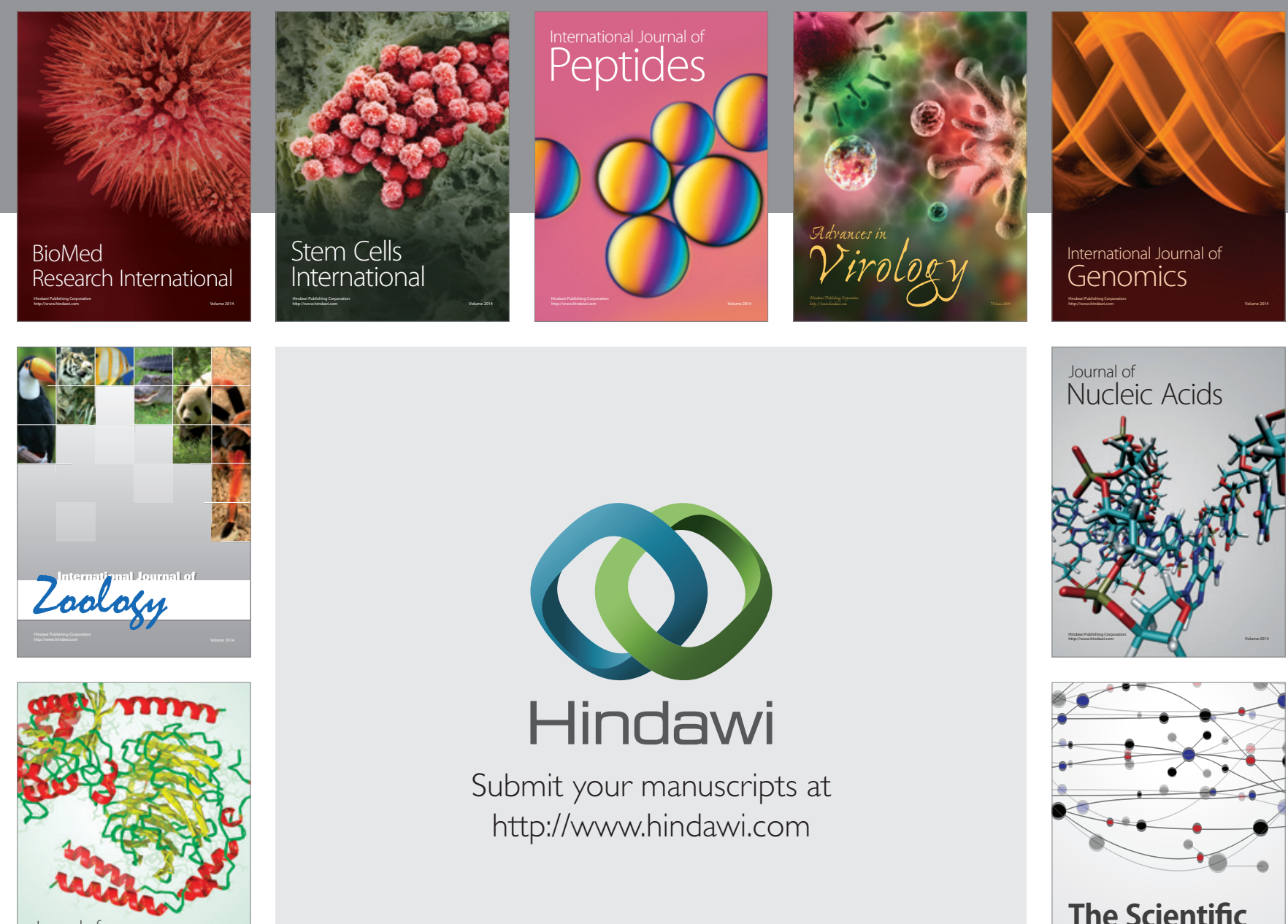

Submit your manuscripts at

http://www.hindawi.com

Journal of
Signal Transduction
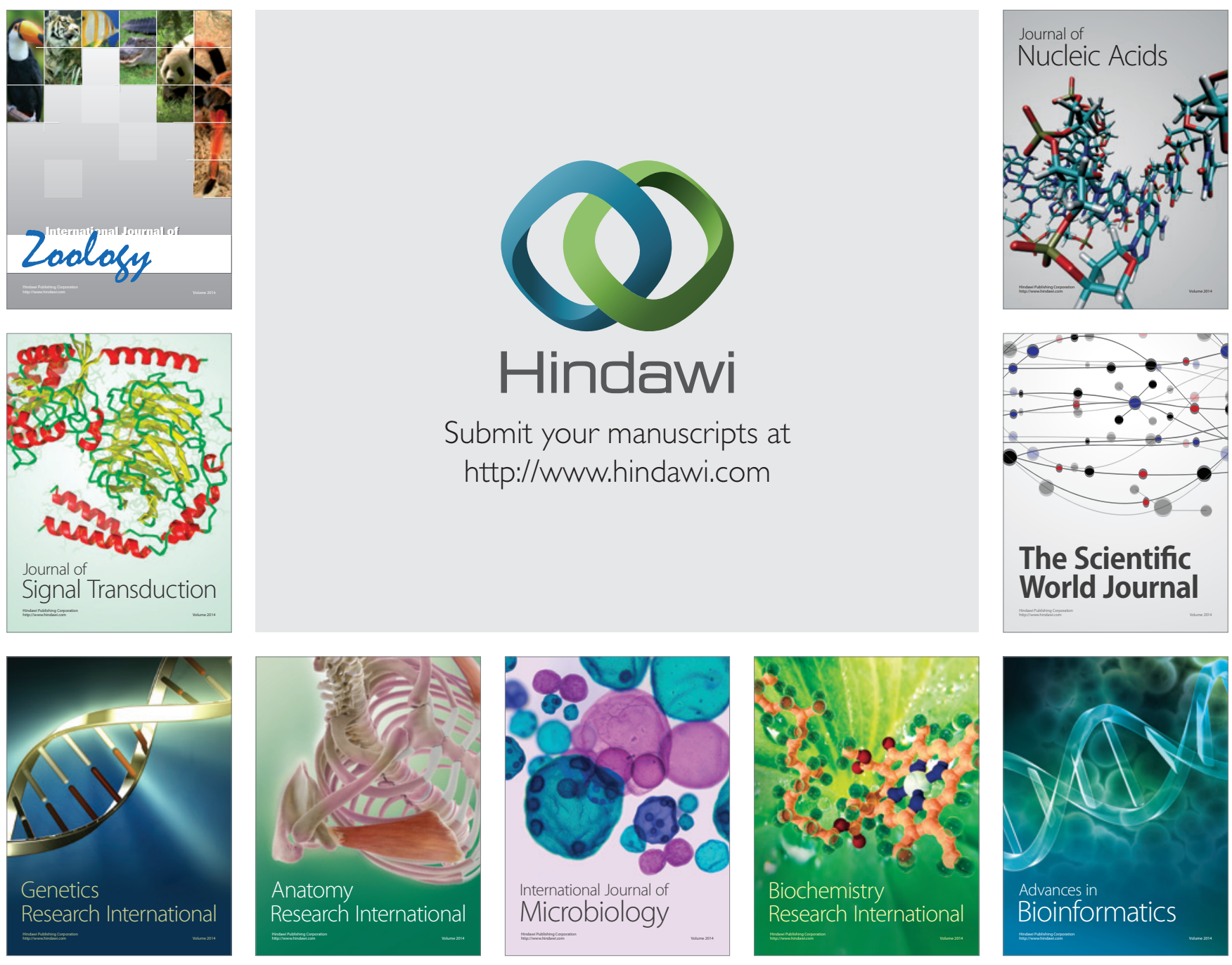

The Scientific World Journal
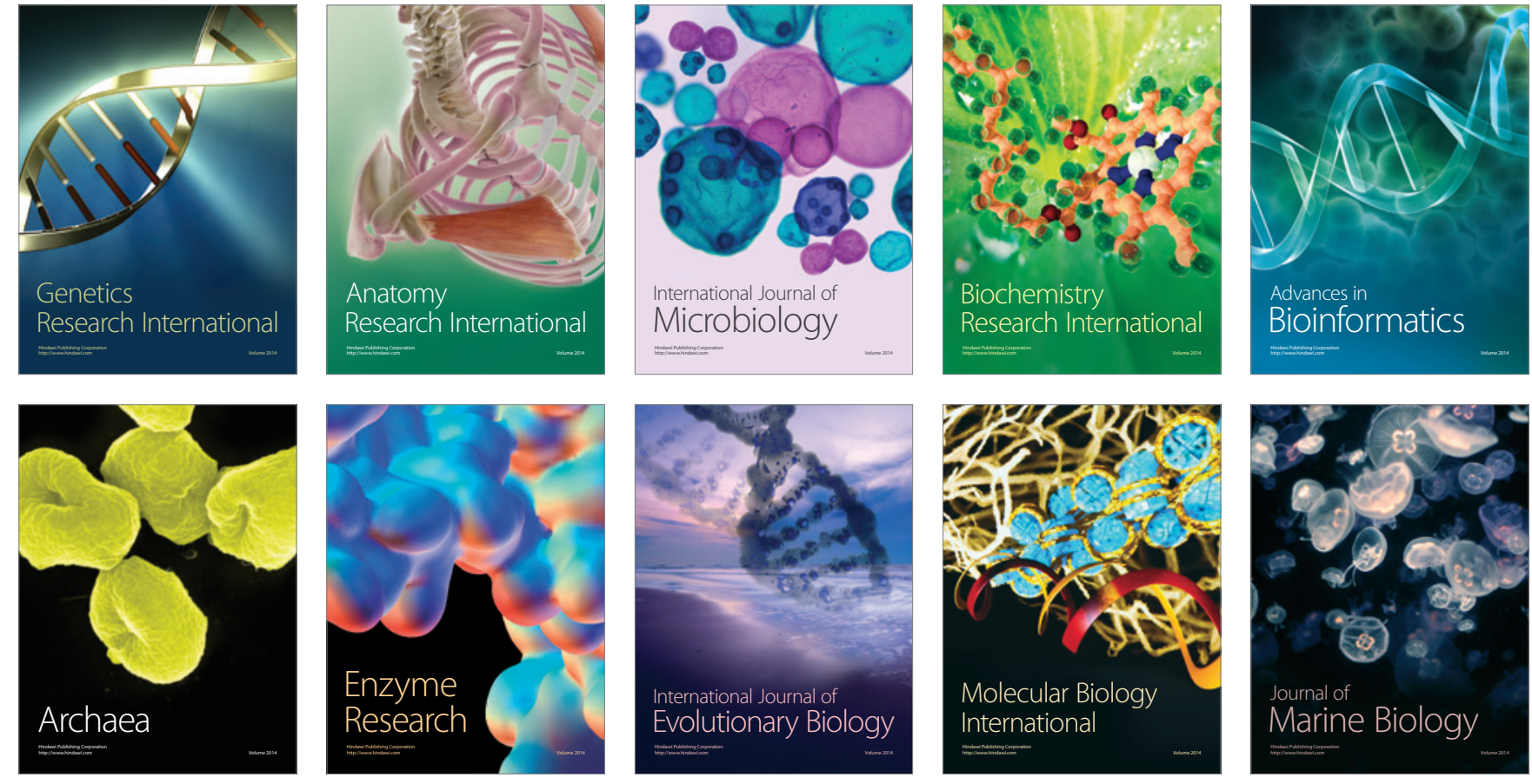